\title{
STRONGLY NEGLIGIBLE SETS IN FRECHET MANIFOLDS
}

\author{
BY R. D. ANDERSON ${ }^{1}$
}

\section{Communicated August 9, 1968}

Let $s$ denote the linear metric space which is the countable infinite product of lines. It is known [1] that $s$ is homeomorphic to Hilbert space $l_{2}$ and, in light of [8] and [10], to all separable infinite-dimensional Fréchet spaces (and therefore, of course, to all such Banach spaces). We define a Fréchet manifold or F-manifold to be a separable metric space which admits an open cover by sets homeomorphic to open subsets of $s$. Banach manifolds, which may be similarly defined, have been studied by a number of authors. From the results cited above it follows that all separable metric Banach manifolds modeled on separable infinite-dimensional Banach spaces are, in fact, $F$ manifolds. Also, clearly, any open subset of an $F$-manifold is an $F$ manifold.

In this paper, we are concerned with homeomorphisms of $F$ manifolds onto dense subsets of themselves. The first result of the type we consider was due to Klee [11], who showed that for any compact set $K$ in $l_{2}, l_{2}$ is homeomorphic to $l_{2} \backslash K$. Recently, there have been a number of results [2], [3], [4], [5], [7], [13], etc., showing that for various types of subsets $K$ of certain linear metric spaces $X$, $X$ is homeomorphic to $X \backslash K$. Bessaga [7] introduced the term "neg-

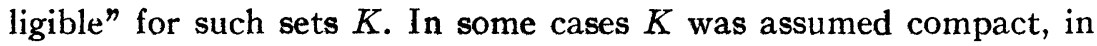
others $\sigma$-compact (i.e. the countable union of compact sets) and in others $K$ was assumed to be the ccuntable union of closed sets of infinite deficiency (i.e. of infinite codimension). Indeed several different geometric methods [2], [3], [5], [7], [11] have been used to establish negligibility in various spaces. The results that $\sigma$-compact subsets of $l_{2}$ and of $s$ are negligible were used in the proofs [1] and [5] that $l_{2}$ is homeomorphic to $s$. Questions of negligibility of subsets in Fréchet and Banach manifolds have also arisen. Where differentiable structures are assumed as for Banach spaces and manifolds and $K$ is assumed closed, Bessaga [7], Corson, Eells and Kuiper [9], Kuiper and Burghelea [12], Moulis [13], Renz [15] and West have investigated conditions under which $X$ and $X \backslash K$ are diffeomorphic,

1 This research was supported in part under NSF Grant GP 6867. A manuscript giving detailed arguments for Theorems $\mathrm{I}$ to $\mathrm{V}$ is in preparation. 
or they have used results of this type in other work. However, the results being announced in this paper are concerned only with homeomorphisms, not with diffeomorphisms.

In [6], Henderson, West, and the author introduced the concept of strong negligibility and characterized the strongly negligible closed subsets of an $F$-manifold. A subset $K$ of a space $X$ is strongly negligible if for any open cover $G$ of $X$ there exists a homeomorphism $h$ of $X$ onto $X \backslash K$ such that $h$ is limited by $G$, i.e., for any $p \in X$ there exists $g \in G$ such that both $p$ and $h(p)$ are elements of $g$.

A similar concept related to the metric of a space is the concept of metric negligibility. A set $K$ in a metric space $X$ is metrically negligible in $X$ if for each $\epsilon>0$, there exists a homeomorphism $h$ of $X$ onto $X \backslash K$ such that $h$ moves no point more than $\epsilon$. Clearly, in a metric space $X$, strong negligibility of a set $K$ implies metric negligibility since we may select an open cover of $X$ of mesh less than $\epsilon$. It is nontrivial, but follows from Theorem $I$ below that, in an $F$-manifold, metric negligibility of a set $K$ implies strong negligibility of $K$.

Following [4], a closed set $K$ has Property $Z$ in a space $X$ if for each nonnull homotopically trivial open set $U$ in $X, U \backslash K$ is nonnull and homotopically trivial. (A set $U$ is homotopically trivial if every map of an $n$-sphere $S^{n}, n \geqq 0$, into $U$ can be extended to a map into $U$ of an $(n+1)$-ball bounded by $S^{n}$.) In a sense, Property $Z$ is "trivial homotopy negligibility." See [9] for a similar point-of-view.

The following theorem is proved in [6].

TheOREM $0 . A$ closed set $K$ in an $F$-manifold $X$ is strongly negligible iff $K$ has Property $Z$.

It should be noted that every compact set in an $F$-manifold $X$ has Property $Z$ in $X$, that every closed set of infinite deficiency in $s$ or in a separable metric Banach space has Property $Z$ in such space, and that every closed set which is a countable union of closed sets with Property $Z$ in an $F$-manifold $X$ has Property $Z$ in $X$.

The principal result of this paper is the following theorem.

Theorem I. $A$ set $K$ in an F-manifold $X$ is strongly negligible (or metrically negligible) in $X$ iff $K$ is a countable union of closed sets with Property $Z$ in $X$.

Theorem I includes, as special cases or easy corollaries, Theorem 0 and many or all of the previous results on negligibility in $F$-manifolds $X$ under homeomorphisms of $X$ onto dense subsets of itself.

The proof of necessity in Theorem $I$ is fairly straightforward. We do not outline it here. 
The proof of sufficiency depends heavily on the canonical compactification of $s$ as the Hilbert cube $I^{\infty}$ in which $s$ is regarded as a product of open intervals and the Hilbert cube is regarded as the product of the closures of the open intervals. Thus $I^{\infty}=\prod_{j>0} I_{j}$ and $s=\prod_{j>0} I_{j}^{0}$ where for each $j>0, I_{j}=[-1,1]$ and $I_{j}^{0}=(-1,1)$. We let $B\left(I^{\infty}\right)$ denote $I^{\infty} \backslash s$. A set $K \subset I^{\infty}$ is an apparent boundary of $I^{\infty}$ if there exists a homeomorphism $h$ of $I^{\infty}$ onto $I^{\infty}$ such that $h(K)=B\left(I^{\infty}\right)$.

In [6], a rather general procedure for reducing certain homeomorphism problems on $F$-manifolds to homeomorphism problems on the Hilbert cube or on $s$ itself is given. The actual homeomorphism theorems on $I^{\infty}$ and $s$ that are needed in [6] can be found in [2], [4], [5]. While we use the general procedures of [6] (with slight modifications) to establish sufficiency in Theorem I, we also use the following new homeomorphism theorem about $I^{\infty}$.

THEOREM II. Let $I^{\infty} \supset K \supset B\left(I^{\infty}\right)$. Then $K$ is an apparent boundary of $I^{\infty}$ iff $K$ is a countable union of closed sets with Property $Z$ in $I^{\infty}$.

In effect, Theorem II characterizes those apparent boundaries of $I^{\infty}$ which contain $B\left(I^{\infty}\right)$.

The sufficiency statement of Theorem II can be used to prove the somewhat stronger Theorem IIA below, which is in a form more readily adaptable for application to $F$-manifolds. An endslice of $I^{\infty}$ is a set $W$ such that for some $i>0, W=\left\{\left(x_{j}\right) \in I^{\infty} \mid x_{i}=1\right.$ (or -1$\left.)\right\}$.

THEOREM IIA. Let $W^{*}$ be a finite union of endslices in $I^{\infty}$, let $\epsilon>0$, and let $K$ be a countable union of closed sets with Property $Z$ in $I^{\infty}$ such that $K \cap W^{*}=\varnothing$. Then there exists a homeomorphism $h$ of $I^{\infty}$ onto $I^{\infty}$ such that $h \mid W^{*}=$ identity, $h(s \backslash K)=s$, and $h$ moves no point more than $\epsilon$.

The "bridge" between Property $Z$ in $s$ and Property $Z$ in $I^{\infty}$ is given by the statement, proved in [4], that for any closed set $K$ in $s$ with Property $Z$ in $s, \mathrm{Cl} K$ in $I^{\infty}$ has Property $Z$ in $I^{\infty}$.

Outline of the Proof of Theorem II. Since an endslice in $I^{\infty}$ has Property $Z$ in $I^{\infty}, B\left(I^{\infty}\right)$ is a countable union of closed sets with Property $Z$ in $I^{\infty}$. Hence necessity follows immediately. We shall reduce the proof of sufficiency to three elementary but nontrivial theorems whose formulations require some additional definitions.

A core is a set $C=\prod_{j>0} J_{j}$ where for each $j>0, J_{j}$ is a closed interval contained in $I_{j}^{0}$. A basic core set $M$ structured on a core $C=\prod_{j>0} J_{j}$ is defined as $M=\left\{\left(x_{j}\right)_{j>0} \in s \mid\right.$ for all but finitely many $\left.j, x_{j} \in J_{j}\right\}$. A core set is a subset of $s$ which is $\sigma$-compact and contains a basic core set. It is easy to verify that a basic core set is a core set.

THEOREM III. Every core set is an apparent boundary of $I^{\infty}$. 
THEOREM IV. For any basic core set $M$ there is a homeomorphism $g$ of $I^{\infty}$ onto $I^{\infty}$ such that $g(M)=B\left(I^{\infty}\right)$, and $g \circ g$ is the identity.

THEOREM V. For any set $K \subset I^{\infty}$ which is the countable union of closed sets with Property $Z$ in $I^{\infty}$, there exist a homeomorphism $f$ of $I^{\infty}$ onto $I^{\infty}$ and a basic core set $M$ such that $f(K) \cap M=\varnothing$, and $f\left(B\left(I^{\infty}\right)\right)=B\left(I^{\infty}\right)$.

Theorems III and IV can be proved by a more delicate argument than that outlined in [4] for the proof of Theorem 11.1 there, together with selected apparatus like that found in [2]. Theorem V can be proved rather routinely from Lemma 6.1 of [4]. We now give a short proof of sufficiency for Theorem II based on Theorems III, IV, and V.

Proof of Sufficiency for Theorem II. Let $K$ be as in the hypothesis. Let $f$ be as in Theorem $\mathrm{V}$, and $g$ be as in Theorem IV. Let, by Theorem III, $h$ carry $g \circ f(K)$ onto $B\left(I^{\infty}\right)$. Then $h \circ g \circ f$ is the desired homeomorphism.

\section{BIBLIOGRAPHY}

1. R. D. Anderson, Hilbert space is homeomorphic to the countable infinite product of lines, Bull. Amer. Math. Soc. 72 (1966), 515-519.

2. - Topological properties of the Hilbert cube and the infinite product of open intervals, Trans. Amer. Math. Soc. 126 (1967), 200-216.

3. - On a theorem of Klee, Proc. Amer. Math. Soc. 17 (1966), 1401-1404.

4. - On topological infinite deficiency, Michigan Math. J. 14 (1967), 365-383.

5. R. D. Anderson and R. H. Bing, A complete elementary proof that Hilbert space is homeomorphic to the countable infinite product of lines, Bull. Amer. Math. Soc. 74 (1968), 771-792.

6. R. D. Anderson, David W. Henderson and James E. West, Negligible subsets of infinite-dimensional manifolds, Compositio Math. (to appear).

7. C. Bessaga, Every infinite-dimensional Hilbert space is diffeomorphic with its unit sphere, Bull. Acad. Polon. Sci. Sér. Sci. Math. Astronom. Phys. 14 (1966), 27-31.

8. C. Bessaga and A. Pelczynski, Some remarks on homeomorphisms of F-spaces, Bull. Acad. Polon. Sci. Sér. Sci. Math. Astronom. Phys. 10 (1962), 265-270.

9. J. Eells and N. Kuiper, Homotopy negligible subsets of infinite-dimensional manifolds, Compositio Math. (to appear).

10. M. I. Kadec, On topological equivalence of separable Banach spaces, Dokl. Akad. Nauk SSSR 167 (1966), 23-25 = Soviet Math Dokl. 7 (1966), 319-322.

11. V. L. Klee, Convex bodies and periodic homeomorphisms in Hilbert space, Trans. Amer. Math. Soc. 74 (1953), 10-43.

12. Nicolaas $H$. Kuiper and Dan Burghelea, Hilbet manifolds, preprint.

13. N. Moulis, Sur les variêtés Hilbertiennes et les fonctions non-dégénerées, preprint.

14. Bor-Luh Lin, Two topological properties of normed linear spaces, Trans. Amer. Math. Soc. 114 (1965), 156-175.

15. Peter Renz, Smooth extensions and extractions in infinite-dimensional Banach spaces, Dissertation, University of Washington, Seattle, Wash., 1968.

Louisiana State University, Baton Rouge, Louisiana 70803 